\title{
$\mathrm{Mn}(I)$ 催化亚胺和炔烃脱氢偶联反应的机理研究
}

\author{
杨一诺张琪石景* 傅尧* \\ (中国科学技术大学化学系 合肥 230026)
}

\begin{abstract}
摘要 近年来随着过渡金属催化剂的发展和广泛使用, $\mathrm{C}-\mathrm{H}$ 键的活化成为了有机合成中的一大热点, 尤其是在构建有 机化合物 $\mathrm{C}-\mathrm{C}$ 键中应用广泛. 作为一种储量较大的廉价催化剂, $\mathrm{Mn}$ 催化的 $\mathrm{C}-\mathrm{H}$ 活化表现出巨大的应用价值和研究 潜力. 我们采用密度泛函理论(DFT), 对 1,4-二氧六环溶液中 $\mathrm{Mn}$ 活化 $\mathrm{C}-\mathrm{H} / \mathrm{N}-\mathrm{H}$ 键实现 [4+2]脱氢环化的反应机理进 行了系统的研究. 我们发现该反应的催化循环包括溴负离子辅助的催化剂引发、炔烃的插入、双键迁移成环、 $\beta$ - $\mathrm{H}$ 消 除释放产物异喹啉以及催化脱氢循环的 $\mathrm{C}-\mathrm{H}$ 键活化等步骤. 旨在深入理解 $\mathrm{Mn}(\mathrm{I})$ 活化 $\mathrm{C}-\mathrm{H}$ 键脱氢气的具体过程, 为 更多 $\mathrm{Mn}$ 催化的 $\mathrm{C}-\mathrm{H}$ 活化反应提供理论依据.
\end{abstract}

关键词 $\mathrm{Mn}$ 催化; 密度泛函理论(DFT); C-H 活化; 反应机理

\section{Mechanism Study of Mn(I) Complex-catalyzed Imines and Alkynes Dehydrogenation Coupling Reaction}

\author{
Yang, Yinuo Zhang, Qi Shi, Jing* Fu, Yao* \\ (Department of Chemistry, University of Science and Technology of China, Hefei 230026)
}

\begin{abstract}
With the development and widespread use of transition metal catalysts, $\mathrm{C}-\mathrm{H}$ activation has become a hot topic in organic synthesis, especially in the construction of $\mathrm{C}-\mathrm{C}$ bond of organic compounds. As an important and cheap catalyst, manganese complex has shown great potential for catalyzing $\mathrm{C}-\mathrm{H}$ activation both in academic and industrial applications. In this paper, the mechanism of manganese-catalyzed dehydrogenative [4+2] annulation by $\mathrm{C}-\mathrm{H} / \mathrm{N}-\mathrm{H}$ activation was investigated systematically with the aid of density functional theory (DFT) calculations in 1,4-dioxane solvent. In detail, we use M06-L/[SDD:6-311+G(d,p)(SMD)]/M06-L/[LANL2DZ:6-31G(d)] to examine the Gibbs free energy, structure and other properties of possible intermediates and transition states in this catalytic cycle. By comprehensive comparison and discussion, we obtained a favorable pathway consisting of five steps: (1) catalyst initiation occurred with the assistance of bromine anion rather than imide to form active catalyst; (2) alkyne inserted into the active catalyst to generate a seven-membered manganacycle after dissociation of a carbon monoxide; (3) double bond migration happened in this seven-membered manganacycle to form a product precursor; (4) the product precursor would dissociate by $\beta$ - $\mathrm{H}$ elimination and generated product isoquinoline and active $\mathrm{Mn}-\mathrm{H}$ complex; (5) the active $\mathrm{Mn}-\mathrm{H}$ complex was subsequently combined with an imine followed by dehydrogenative $\mathrm{C}-\mathrm{H}$ activation to complete the whole catalytic cycle. In this context, the reason for the highly atom-economical $\mathrm{C}-\mathrm{H}$ activation by direct dehydrogenation (eliminates the necessity for oxidants or additives) has been clarified by this mechanism. The present study was aimed at further understanding of Mn(I)-catalyzed dehydrogenative $\mathrm{C}-\mathrm{H}$ activation, and provided more theoretical basis for future more $\mathrm{Mn}$-catalyzed $\mathrm{C}-\mathrm{H}$ activation.

Keywords manganese-catalyzed; density functional theory (DFT); C-H activation; mechanism
\end{abstract}

\section{1 引言}

过渡金属催化的 $\mathrm{C}-\mathrm{H}$ 键活化具有较高的原子经济 性和合成效率, 是构建 $\mathrm{C}-\mathrm{C}$ 键骨架的重要策略, 近年 来受到了十分广泛的关注 ${ }^{[1]}$. 目前, 有关 $\mathrm{C}-\mathrm{H}$ 键活化 的研究主要集中在 $\mathrm{Rh} 、 \mathrm{Ru} 、 \mathrm{Pd} 、 \mathrm{Ir} 、 \mathrm{Cu}$ 等过渡金属催 化剂上 ${ }^{[2]}$, 而对价格低廉且存储量较大的 $\mathrm{Mn}$ 催化剂研
究较少 ${ }^{[3]}$. 近几年, $\mathrm{Mn}$ 催化的 $\mathrm{C}-\mathrm{H}$ 键活化以其卓越的 应用潜能引起了化学工作者的重点关注. 2007 年 Kuninobu 和 Takai 等 ${ }^{[4]}$ 在 $\left[\mathrm{MnBr}(\mathrm{CO})_{5}\right]$ 催化剂条件下实 现了醛插入芳烃 $\mathrm{C}-\mathrm{H}$ 键的反应; 2013 年 Chen 和 Wang 等 ${ }^{[5]}$ 报道了 $\left[\mathrm{MnBr}(\mathrm{CO})_{5}\right]$ 催化末端炔和芳烃 $\mathrm{C}-\mathrm{H}$ 键的 加成反应. 然而, 这些反应均无法完全实现原子经济性 下的高效转化. 最近, Wang 小组 ${ }^{[6]}$ 使用 1,4 -二氧六环作

\footnotetext{
*E-mail: fuyao@ustc.edu.cn; shijing@ustc.edu.cn

Received November 21, 2015; published March 18, 2016.

Supporting information for this article is available free of charge via the Internet at http://sioc-journal.cn

Project supported by the National Natural Science Foundation of China (Nos. 21325208, 21172209, 21361140372, 21202006), 973 Program (No. 2012CB215306), Fundamental Research Funds for the Central Universities (Nos. WK2060190025, WK2060190040, FRF-TP-14-015A2) and Science Foundation of The Chinese Academy of Sciences (No. KJCX2-EW-J02).

项目受国家自然科学基金(Nos. 21325208，21172209，21361140372，21202006)、973 计划(No. 2012CB215306)、中央高校基础研究经费(Nos. WK2060190025, WK2060190040, FRF-TP-14-015A2)和中国科学院基金(No. KJCX2-EW-J02)资助.
} 
为溶剂, 在 $\left[\mathrm{MnBr}(\mathrm{CO})_{5}\right]$ 催化下通过活化 $\mathrm{C}-\mathrm{H} / \mathrm{N}-\mathrm{H}$ 键 实现亚胺和炔烃 $[4+2]$ 脱氢环化生成异喹啉的反应(Eq. 1). 该反应在 $\mathrm{Mn}$ 催化剂的条件下可以直接通过脱氢气 实现 $\mathrm{C}-\mathrm{H}$ 活化 $/ \mathrm{C}-\mathrm{C}$ 偶联, 不再需要氧化剂和添加剂, 实现了真正意义上的原子经济性.

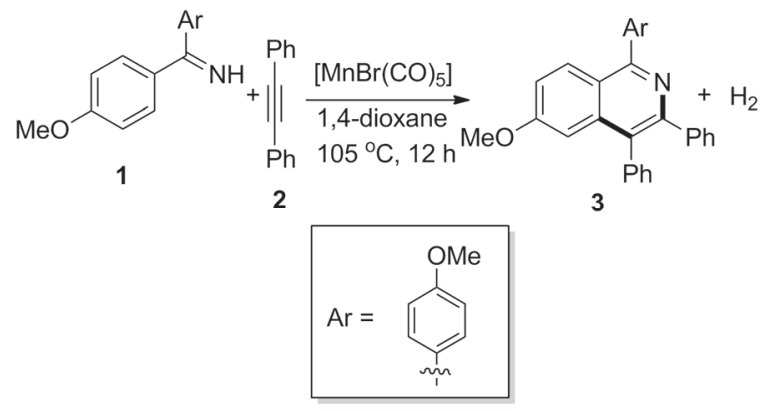

对于这个重要的脱氢偶联反应, Wang 小组 ${ }^{[6]}$ 提出了 一个可能的机理假设. 如 Scheme 1 所示, 首先催化剂 $\left[\mathrm{MnBr}(\mathrm{CO})_{5}\right]$ 与亚胺 $(\mathbf{1})$ 反应得到五元环锰络合物 $\mathbf{A}$. 机 理实验中 1 与 $\mathrm{Mn}$ 催化剂的定量实验能够分离得到 $\mathbf{A}$, 且 $\mathbf{A}$ 可以催化反应的进行, 因此他们推断 $\mathbf{A}$ 是催化循环 的活性中间体. 随后 $\mathbf{A}$ 与炔烃络合形成中间体 $\mathbf{B}$. B 经历 炔烃插入生成七元环 Mn 络合物 $\mathbf{C}$. $\mathbf{C}$ 解离得到异喹啉 产物 3 和锰氢络合物 D. D 再与 1 络合并活化 1 中 $\mathrm{C}-\mathrm{H}$ 键, 脱去氢气重新得到络合物 $\mathbf{A}$, 进而完成整个催化循 环.

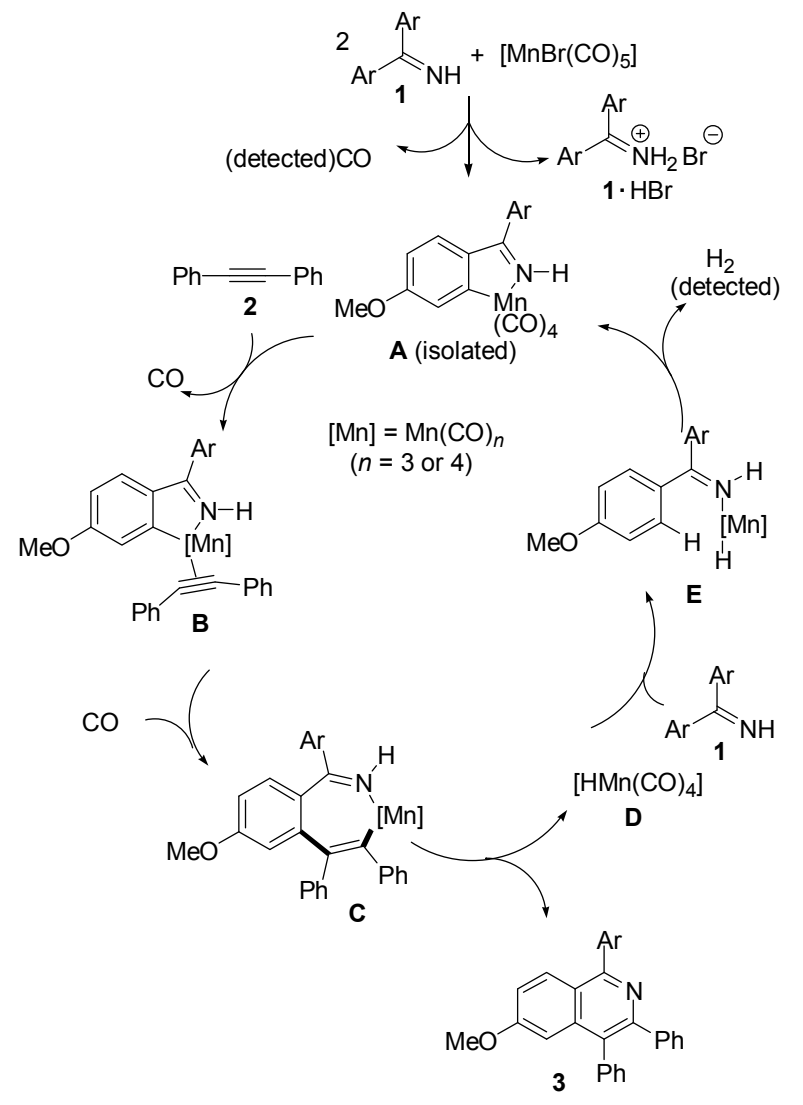

该机理有益于增进我们对 $\mathrm{Mn}$ 催化 $\mathrm{C}-\mathrm{H}$ 键活化官 能团化的理解, 然而其细节仍有待推敲. 例如, 七元环 锰络合物 $\mathbf{C}$ 生成产物 $\mathbf{3}$ 的具体过程, 锰氢络合物 $\mathbf{E}$ 经历 $\mathrm{C}-\mathrm{H}$ 活化的详细机理等仍然不清楚. 为了解决以上问 题, 我们利用密度泛函理论(DFT)方法对反应机理进行 了系统研究. 通过 DFT 计算, 我们得到了该反应最优的 机理路径, 为 $\mathrm{Mn}$ 催化 $\mathrm{C}-\mathrm{H}$ 键活化的反应提供了一定 的理论基础.

\section{2 结果与讨论}

\section{1 催化剂的引发}

Wang 小组 ${ }^{[6]}$ 在反应中通过定量分步实验验证了五 元环锰络合物 $\mathbf{A}$ 是催化反应必经的一个中间体, 因此我 们首先研究亚胺反应物 $\mathbf{1}$ 到活性络合物 $\mathbf{A}$ 的催化剂引发 过程. 结合 Wang 小组 ${ }^{[7]}$ 此前对 $\mathrm{Mn}$ 络合物活化 $\mathrm{C}-\mathrm{H}$ 键 的机理研究, 我们推测该过程的机理如图 1 所示: 亚胺 反应物 1 与 $\mathrm{Mn}$ 催化剂络合得到中间体 Mn-0, Mn-0 在 Lewis 碱的辅助下发生 C-H 键的活化生成络合物 A. 由 于该过程中溴负离子和亚胺反应物 $\mathbf{1}$ 均可作为 Lewis 碱, 因此我们考虑了两种 $\mathrm{C}-\mathrm{H}$ 键活化的过程. 由能量曲线 可知, 溴负离子辅助的 $\mathrm{C}-\mathrm{H}$ 键活化能垒为 +35.8 $\mathrm{kcal} / \mathrm{mol}(\mathbf{1} \rightarrow \mathbf{T S a})$, 比亚胺反应物 $\mathbf{1}$ 辅助的能垒 $(+38.7$ $\mathrm{kcal} / \mathrm{mol}, \mathbf{1} \rightarrow \mathbf{T S b}$ )低 $2.9 \mathrm{kcal} / \mathrm{mol}$, 这是由于溴负离子碱 性更强且与反应物的位阻作用较小. 由于从 $\mathbf{1}$ 到 $\mathbf{A}$ 的引 发过程不参与催化循环圈, 尽管能垒较高 $(+35.8$ $\mathrm{kcal} / \mathrm{mol}$ ), 但是只需发生微量反应, 得到很少量 $\mathbf{A}$, 即 可不断的促进催化循环圈中的反应. 因此在 $2.2 \sim 2.5$ 节 中计算反应能垒时均以 $\mathbf{A}$ 为起点.

\section{2 炔烃的插入}

由络合物 $\mathbf{A}$ 开始，反应将经历炔烃插入形成七元环 络合物. 因为 $\mathbf{A}$ 中的 $\mathrm{Mn}$ 中心已配位饱和, 所以 $\mathrm{Mn}$ 需 要解离一个配体得到一个空余的络合位点才能与炔烃 $\mathbf{2}$ 络合. 如图 2 所示, 我们首先考虑 $\mathbf{A}$ 中的亚胺基团从 $\mathrm{Mn}$ 中心解离形成中间体 Int2-x, 但是这个过程需要吸 热 $37.2 \mathrm{kcal} / \mathrm{mol}$ (因为烯烃共轭环的扭曲), 因此排除该 机理. 此外, 我们还考察了 $\mathrm{Mn}$ 中心失去 $\mathrm{CO}$ 与炔烃配位 的可能性. 如图 2 所示, $\mathbf{A}$ 失去一分子 $\mathrm{CO}$ 形成五配位中 间体 Int1, 然后与炔烃 2 络合生成炔烃插入前体 Int2. Int2 随后经历四元环的炔烃插入过渡态 TS1 得到七元 环锰中间体 Int3. 炔烃插入能垒为 $+35.6 \mathrm{kcal} / \mathrm{mol}$ $(\mathbf{A} \rightarrow \mathbf{T S 1})$, 该过程吸热 $12.3 \mathrm{kcal} / \mathrm{mol}(\mathbf{A} \rightarrow \mathbf{I n t} 3)$.

\section{3 异喹啉产物 3 和锰氢络合物的生成}

如图 3 所示，炔烃插入所形成的七元环中间体 Int3 的 $\mathrm{Mn}$ 中心配位不饱和, 因而会与一分子 $\mathrm{CO}$ 重新络合 得到热力学更稳定的 Int4, 该过程放热 $18.0 \mathrm{kcal} / \mathrm{mol}$. 对于 Int4 解离得到异喹啉产物 3 和锰氢络合物的过程,

图式 1 Wang 小组提出的反应机理

Scheme 1 The mechanism proposed by Wang's group 
a)
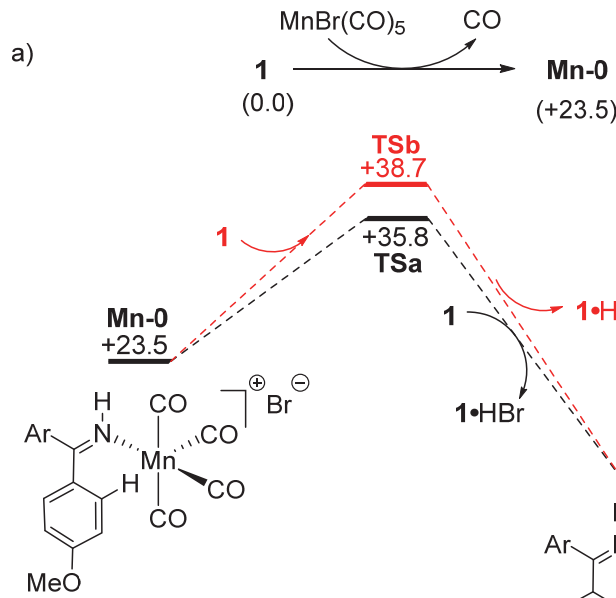

$(+23.5)$

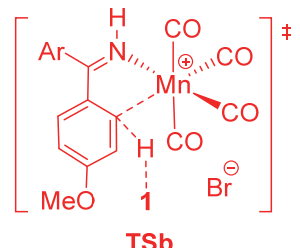

TSb

Sa

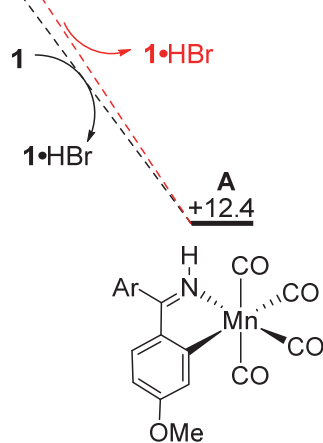

b)

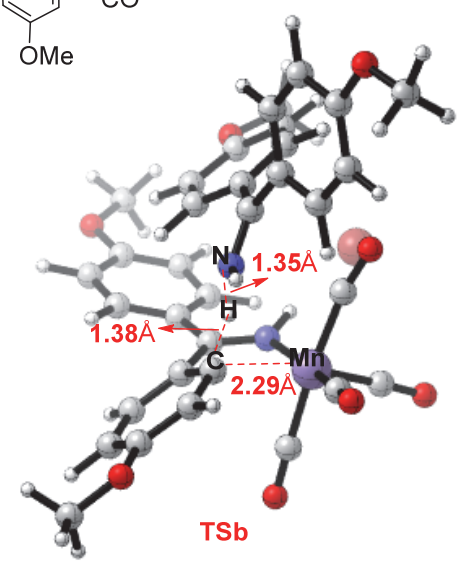

图 1 (a)催化剂引发生成络合物 $\mathbf{A}$ 的两种可能机理; (b)过渡态 TSa/TSb 的空间结构; 本文中所有的能量均为吉布斯自由能, 单位为 $\mathrm{kcal} / \mathrm{mol}$ Figure 1 (a) Two possible mechanisms of complex A's generation in catalyst initiation; (b) The spatial structures of TSa/TSb; Energies here are all Gibbs free energies in kcal/mol

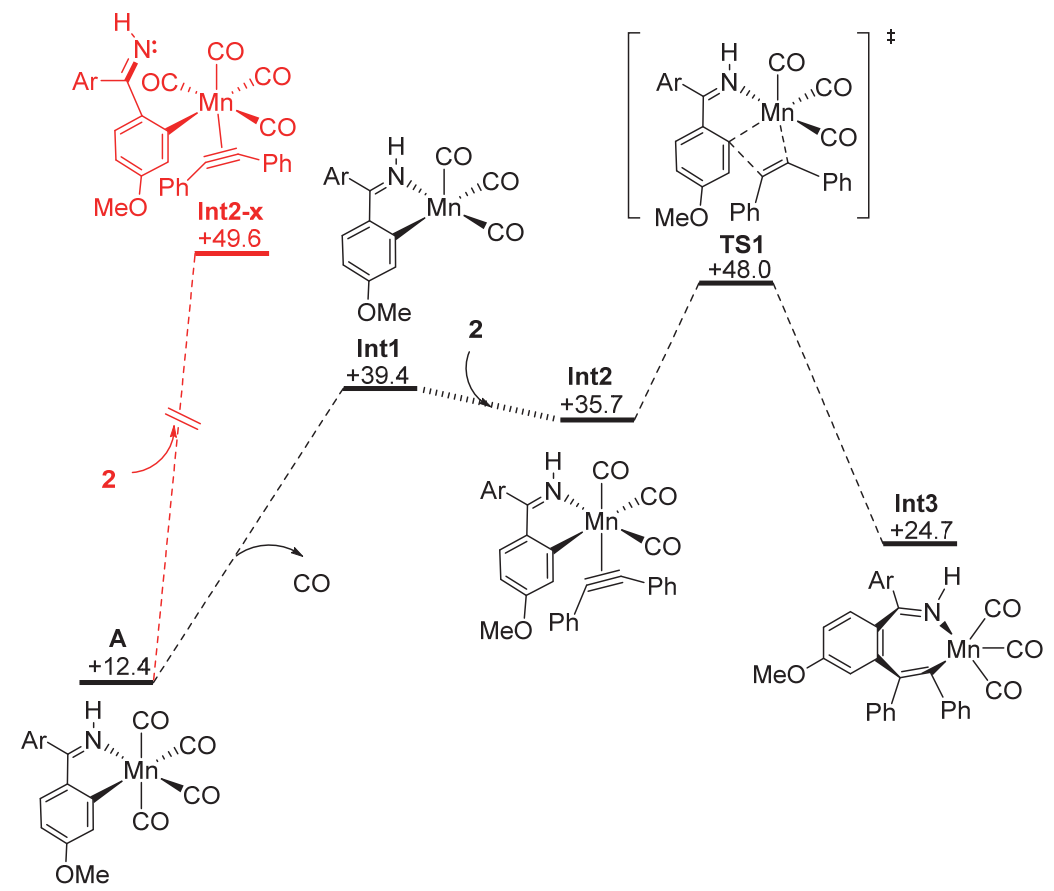

图 2 炔烃插入的可能机理

Figure 2 Possible mechanisms of alkyne insertion 

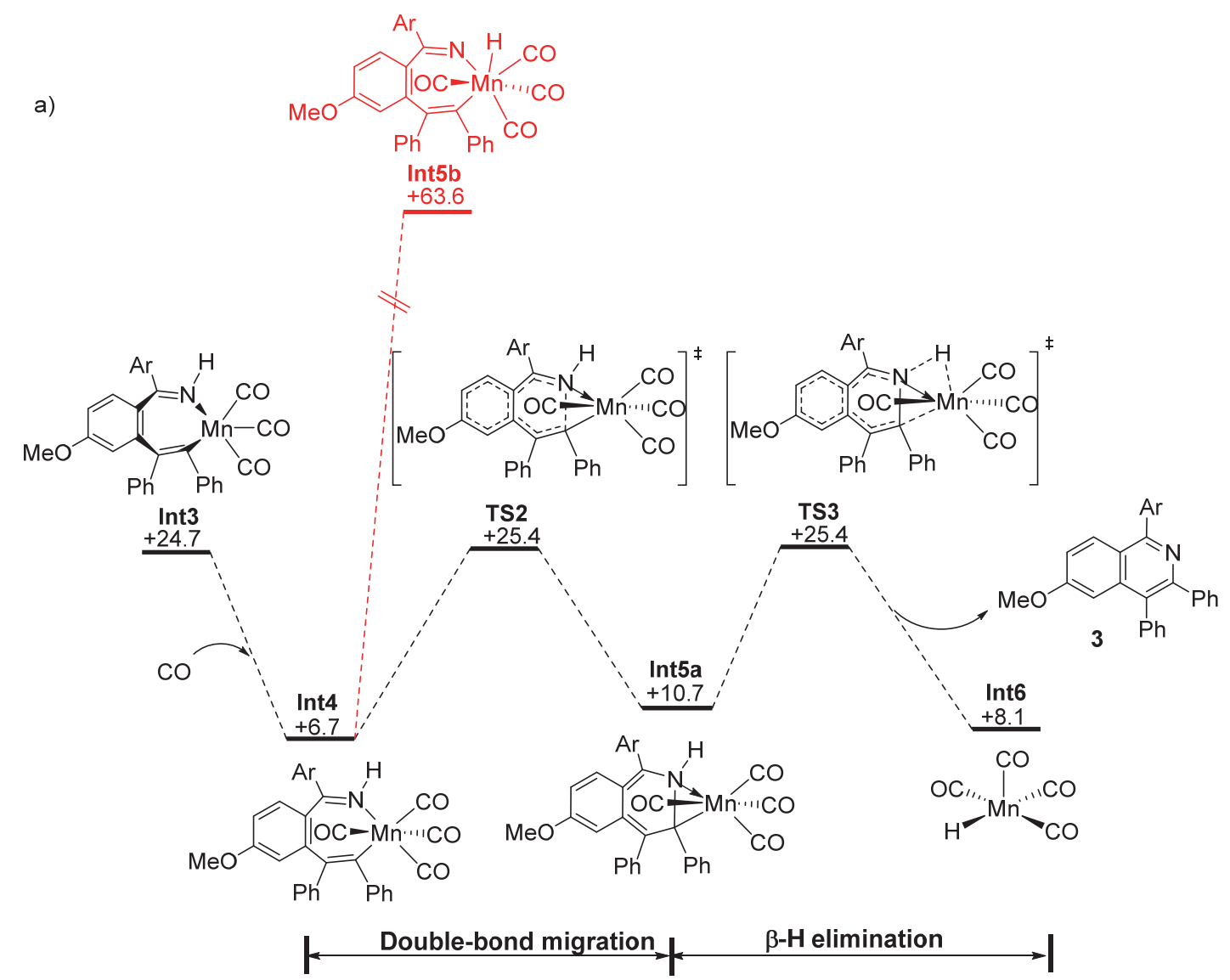

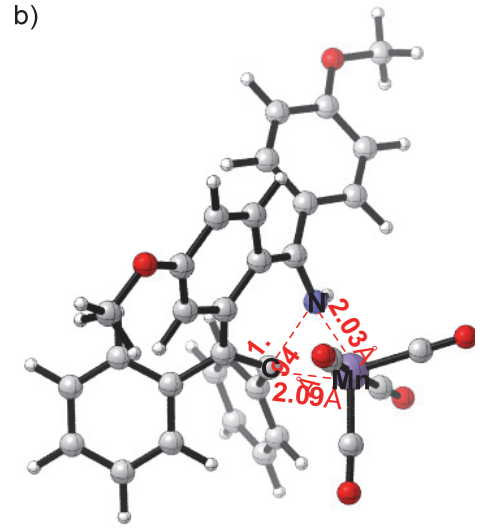

TS2

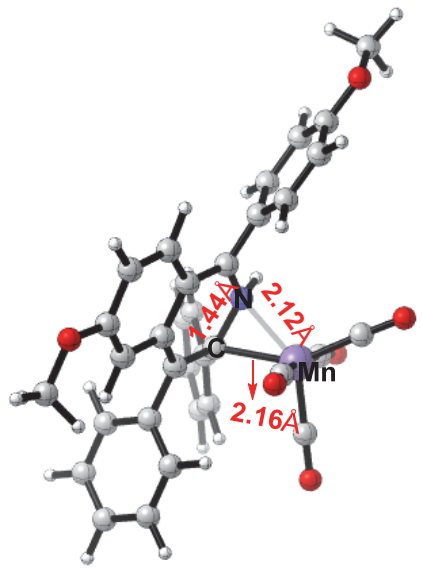

Int5a

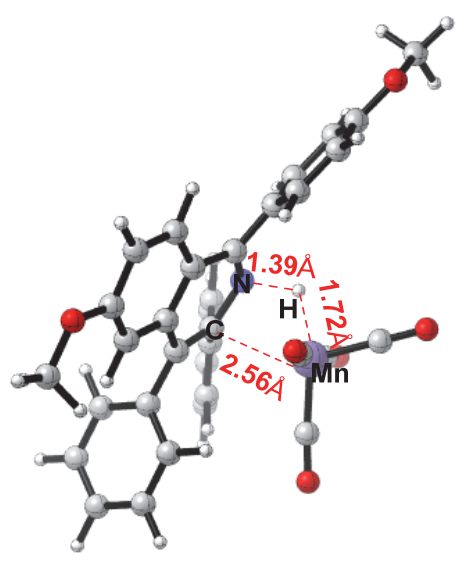

TS3

图 3 (a) 七元环锰络合物 Int4 解离的可能机理; (b)过渡态 TS2/TS3 和中间体 Int5a 的空间结构

Figure 3 (a) Possible decomposition mechanisms of seven-membered manganacycle Int4; (b) The optimized structures of TS2/TS3/Int5a

我们分别考察 Wang 小组提出的两种可能机理: a) $\mathrm{N}-\mathrm{H}$ 键氧化加成/ $\mathrm{C}-\mathrm{N}$ 键还原消除机理, b) $\mathrm{C}-\mathrm{Mn}$ 键 $/ \mathrm{N}-\mathrm{H}$ 键的复分解机理. 对于 $\mathrm{N}-\mathrm{H}$ 键氧化加成/ $\mathrm{C}-\mathrm{N}$ 键还原 消除机理, 由于 $\mathrm{N}-\mathrm{H}$ 键氧化加成得到 $\mathrm{Mn}(\mathrm{III})$ 中间体 Int5b 的能垒高达 $+56.9 \mathrm{kcal} / \mathrm{mol}$, 因此排除该过程. 对 于 $\mathrm{C}-\mathrm{Mn}$ 键/ $\mathrm{N}-\mathrm{H}$ 键的复分解机理, 我们发现复分解 过程不是经历协同的四元环过渡态, 而是经历一个分步 过程. 七元环中间体 Int4 通过双键的转移经历过渡态 TS2 得到 C- $\mathrm{N}$ 成键的稳定六元环中间体 Int5a, 双键迁 移过程需要克服 $18.7 \mathrm{kcal} / \mathrm{mol}$ 的能垒. Int5a 再通过 $\beta-\mathrm{H}$
消除过渡态 TS3 形成异喹啉产物 3 和镍氢中间体 Int6, $\beta-\mathrm{H}$ 消除过程需要克服 $18.7 \mathrm{kcal} / \mathrm{mol}$ 的能垒. 比较上述 两种机理, 我们发现, Int4 转化为 Int6 先后经历双键迁 移和 $\beta-\mathrm{H}$ 消除两个步骤, 其中 $\mathrm{Mn}(\mathrm{I})$ 价态保持不变, 芳 环的存在是双键迁移发生的前提条件.

\section{4 活性催化剂的脱氢再生过程}

随着异喹啉产物 3 的生成, 解离出的锰氢络合物 Int6 再与亚胺反应物 1 经历脱氢过程恢复活性催化剂. 如图 4 所示, Int6 络合一分子亚胺反应物 $\mathbf{1}$ 得到中间体 
a)<smiles>COc1ccc(C(=O)NN=C2CC3CCC2C3)c([N+]([O-])([O-])[O-])c1</smiles>

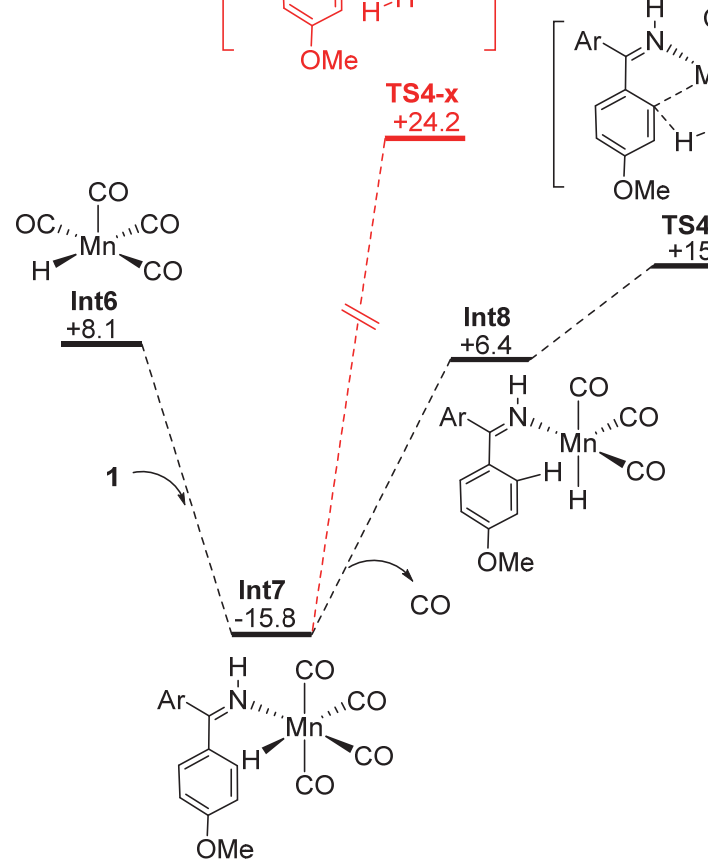<smiles>[H][Y10]1(C(=O)O)CN=CCC1CCOC</smiles>

TS4

b)

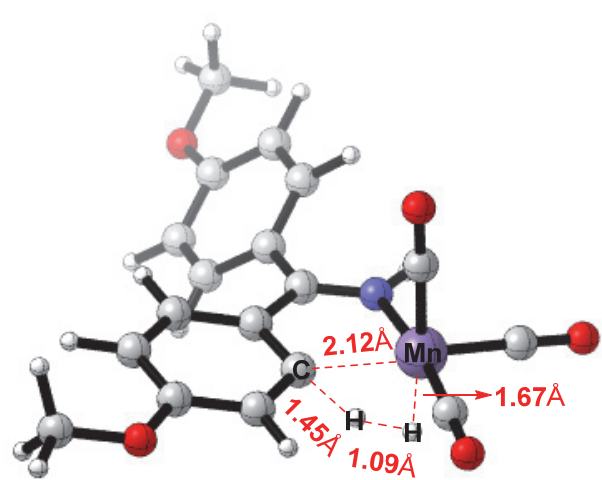

TS4
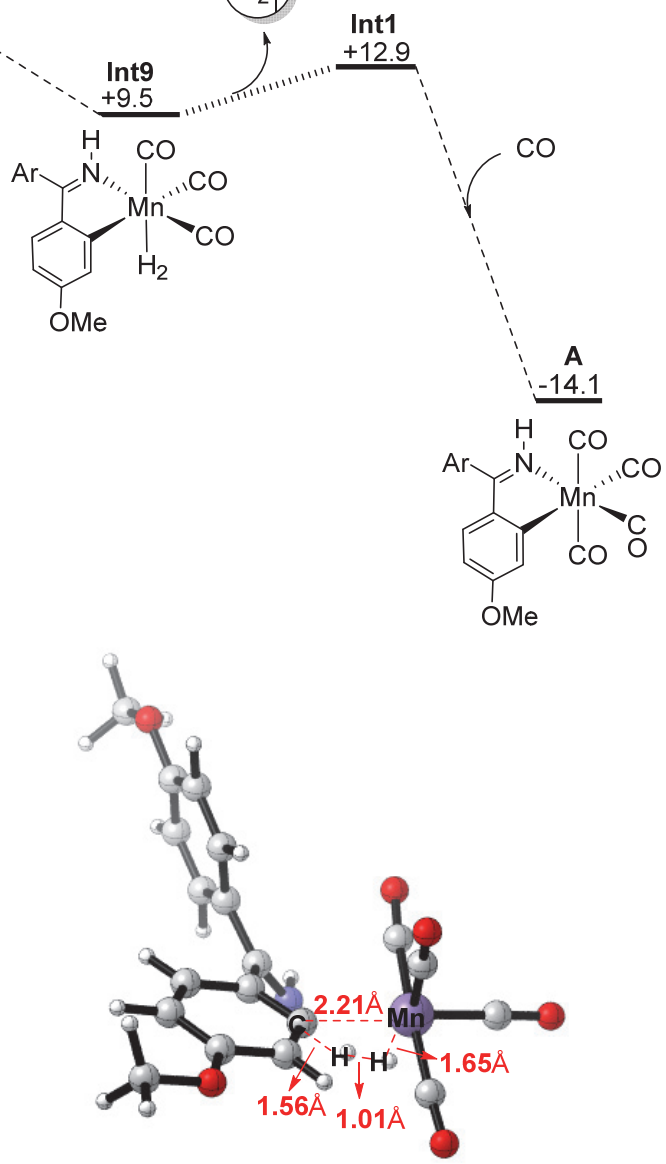

TS4-x

图 4 (a) C-H 活化脱氢的可能机理; (b)过渡态 TS4 和 TS4-x 的空间结构

Figure 4 (a) Possible mechanisms of $\mathrm{C}-\mathrm{H}$ activation/dehydrogenation; (b) The spatial structures of TS4/TS4-x

Int7，这是一个富电子配体络合不饱和炔烃的过程，因 此 Int7 的能量很低, 该过程放热 $23.9 \mathrm{kcal} / \mathrm{mol}$. 由于随 后的 $\mathrm{C}-\mathrm{H} / \mathrm{Mn}-\mathrm{H}$ 协同脱氢气时 $\mathrm{Mn}$ 需要提供一个络合 位点给芳环, 而 Int7 的 Mn 中心已配位饱和, 因此 Int7 需要解离掉一个配体. 我们首先研究 Int7 的 Mn 中心解 离亚胺配体再协同脱氢的可能性. 但是这个脱氢过渡态 TS4-x 需要克服 $40.0 \mathrm{kcal} / \mathrm{mol}$ 的能垒(因为烯烃共轭环的 扭曲), 因此该过程被排除. 此外, 我们还考察了 Int7 的 $\mathrm{Mn}$ 中心先解离 $\mathrm{CO}$ 再 $\mathrm{C}-\mathrm{H}$ 活化脱氢的可能性. Int7 解 离一分子 $\mathrm{CO}$ 得到 Int8, Int8 再经历协同脱氢的过渡态 TS4 得到 $\mathrm{C}-\mathrm{H}$ 键断裂/C-Mn 键形成的中间体 Int9, Int9 解离氢气得到中间体 Int1, Int1 再与 CO 络合即可
再次得到 $\mathbf{A}$. 从 $\mathbf{I n t 7}$ 到 $\mathbf{A}$, 能垒为 $30.8 \mathrm{kcal} / \mathrm{mol}$, 吸热 $1.7 \mathrm{kcal} / \mathrm{mol}$. 根据以上计算结果, 我们得到和 Wang 小 组相同的结论：中间体 $\mathbf{A}$ 是该反应的活性催化剂.

\section{5 整个催化循环过程}

为了更直观的理解反应，我们将整个催化循环整理 在图 5 中. 活性催化剂 $\mathbf{A}$ 经历脱 $\mathrm{CO}$ 和炔烃插入形成七 元环锰中间体 Int3/Int4, Int4 在此经历双键迁移 $($ Int4 $\rightarrow$ Int5a) 和 $\beta-\mathrm{H}$ 消除 $($ Int5a $\rightarrow$ Int6) 得到锰氢络合物 Int6 并释放出异喹啉产物 3, 最后 Int6 活化亚胺 $\mathrm{C}-\mathrm{H}$ 键释放氢气并再次络合 $\mathrm{CO}$ 重新形成活性催化剂 A. 它 们的能垒分别为 $35.6,18.7,18.7,30.8 \mathrm{kcal} / \mathrm{mol}$. 


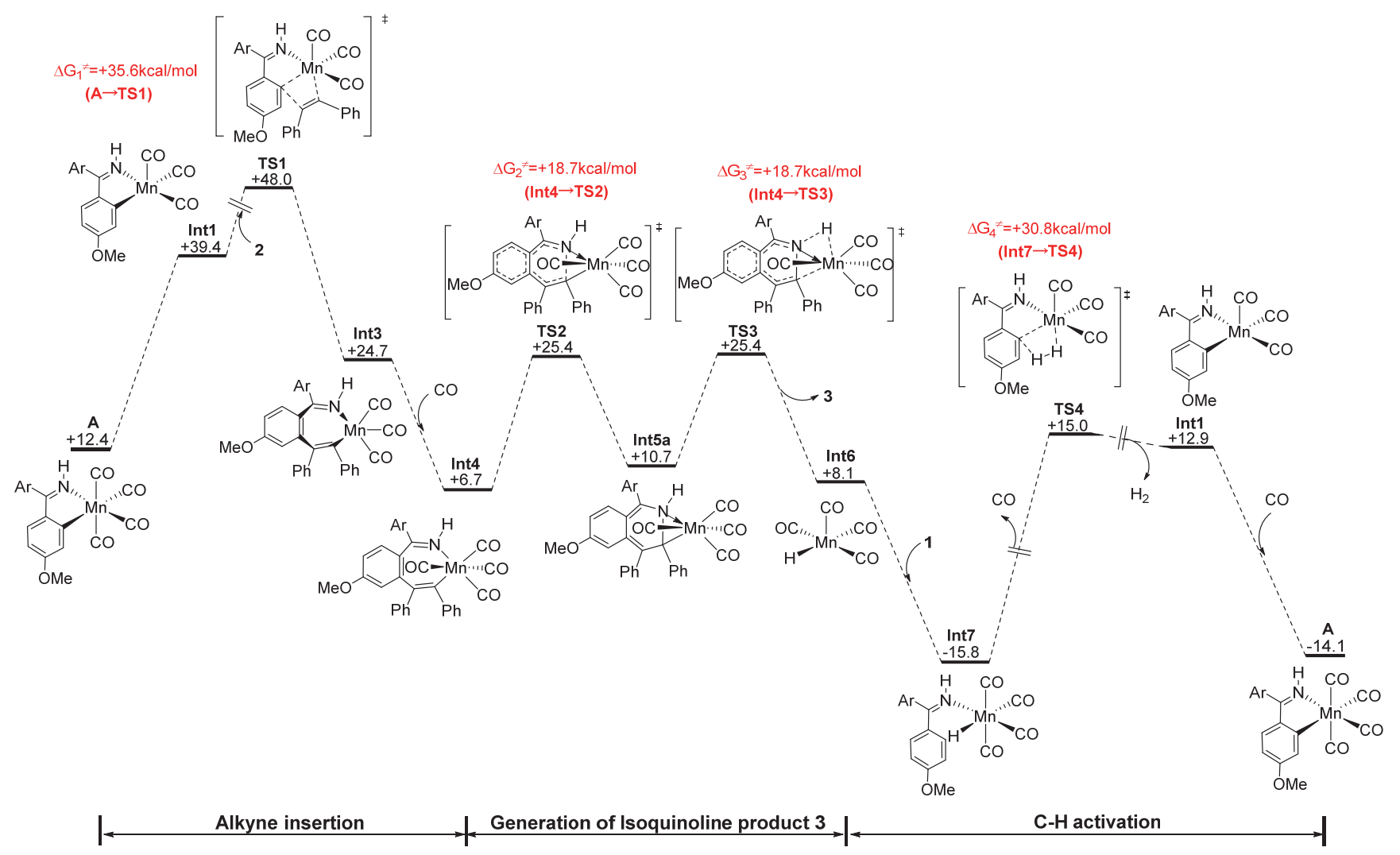

图 5 整个反应的催化循环圈

Figure 5 The whole catalytic cycle of the reaction

\section{3 结论}

本文中, 我们运用 DFT 方法研究 $\mathrm{Mn}(\mathrm{I})$ 催化的亚胺 与炔烃脱氢偶联反应的机理. 通过系统的计算和分析, 我们有以下几点发现: (1)在催化剂引发的过程中, 澳负 离子辅助脱质子的机理比反应物亚胺辅助的机理更优 势, 这与前人提出的机理略有不同 ${ }^{[7]} ;(2)$ 在生成异喹啉 产物 3 的机理中, 中间体 Int4 可以直接经历先双键转移 成环、再 $\beta-\mathrm{H}$ 消除生成产物的过程; (3)在 $\mathrm{C}-\mathrm{H}$ 活化脱 氢的过程中, 先解离一分子 $\mathrm{CO}$ 再协同脱氢的过程更优 势，因此该反应中的活性催化剂是中间体 A. 综上所述, 我们总结该反应经历了溴负离子辅助的催化剂引发、炔 烃的插入、双键迁移成环、 $\beta-\mathrm{H}$ 消除得到产物 $3 、 \mathrm{C}-\mathrm{H}$ 活化脱氢的催化循环过程. 我们对每一步的细节进行了 详细研究, 确定了优势机理, 并且与实验现象相符. 希 望本文的机理研究能够为类似过渡金属催化的 $\mathrm{C}-\mathrm{H}$ 活 化反应提供一定的理论基础.

\section{4 计算方法}

所有的 DFT 计算均在 Gaussian $09^{[8]}$ 中进行. 本文采 用 M06-L 方法 ${ }^{[9]}$ 对反应中涉及的所有反应物、中间体、 过渡态和产物的几何构型进行了气相优化. 优化时, 对 $\mathrm{Mn}$ 元素采用 LANL2DZ 赝势基组 ${ }^{[10]}$, 对 $\mathrm{C} 、 \mathrm{H} 、 \mathrm{O} 、 \mathrm{~N}$ 等元素采用 6-31G(d)全电子基组. 通过频率分析计算,
确定得到的中间体是表征势能面上的极小值稳定点(零 虚频), 得到的过渡态有且只有一个正确振动方向的虚 频. 通过过渡态的内禀反应坐标计算(IRC $)^{[1]}$ 来确认这 种结构确实连接相关的两个极小值.

考虑到溶剂的影响, 本文采用 M06-L 方法在 SMD 溶剂化模型 ${ }^{[12]}$ 下对优化后的所有结构进行了单点能量 计算，根据实验条件选用 1,4-二氧六环作为溶剂. 计算 时, 对 $\mathrm{Mn}$ 元素采用 SDD 赝势基组 ${ }^{[13]}$ ，对 $\mathrm{C} 、 \mathrm{H} 、 \mathrm{O} 、 \mathrm{~N}$ 等元素采用 6-311+G(d,p)全电子基组. 文中所有的吉 布斯自由能为气相吉布斯自由能热力学修正和溶剂化 全电子能之和 ${ }^{[14]}$. 考虑到熵效应的影响, 我们参考前人 的工作经验 ${ }^{[15]}$, 对组分发生变化的基元步骤(1:2 或 2:1) 的相对自由能变化进行了 +2.6 (或 -2.6$) \mathrm{kcal} / \mathrm{mol}$ 的校 正.

\section{致谢}

感谢深圳以及中国科学技术大学的国家超级计算 中心提供的计算资源.

\section{References}

[1] (a) Wencel-Delord, J.; Glorious, F. Nat. Chem. 2013, 5, 369. (b) Ackermann, L. Chem. Rev. 2011, 111, 1315. (c) Yeung, C. S.; Dong, V. M. Chem. Rev. 2011, 111, 1215. (d) Gutekunst, W. R.; Baran, P. S. Chem. Soc. Rev. 2011, 40, 1976. (e) Iwai, T.; Sawamura, M. ACS Catal. 2015, 5, 5031. (f) Segawa, Y.; Maekawa, T.; Itami, K. Angew. 
Chem., Int. Ed. 2015, 54, 66. (g) Topczewski, J. J.; Sanford, M. S. Chem. Sci. 2015, 6, 70. (h) Liao, G.; Shi, B. Acta Chim. Sinica 2015, 73, 1283. (廖港, 史炳锋, 化学学报, 2015, 73, 1283.) (i) Zhou, L.; Lu, W. Acta Chim. Sinica 2015, 73, 1250. (周励宏, 陆文军, 化学 学报, 2015, 73, 1250.) (j) Zhao, J.; Zhang, Q. Acta Chim. Sinica 2015, 73, 1235. (赵金钵, 张前, 化学学报, 2015, 73, 1235.) (k) Shang, X.; Liu, Z. Acta Chim. Sinica 2015, 73, 1275. (尚䈗洁, 柳忠 全, 化学学报, 2015, 73, 1275.) (1) Pan, F.; Shi, Z. Acta Chim. Sinica 2012, 70, 1679. (潘菲, 施章杰, 化学学报, 2012, 70, 1679.) (m) Zhao, H.; Sun, H.; Wang, L.; Li, X. Acta Chim. Sinica 2015, 73, 1307. (n) Qiu, H.; Zhang, D.; Liu, S.; Qiu, L.; Zhou, J.; Qian, Y.; Zhai, C.; Hu, W. Acta Chim. Sinica 2012, 70, 2484. (邱晃, 张丹, 刘顺英, 邱林, 周俊, 钱宇, 翟昌伟, 胡文浩, 化学学报, 2012, 70, 2484.)

[2] (a) Ye, B.; Cramer, N. Acc. Chem. Res. 2015, 48, 1308. (b) Arockiam, P. B.; Bruneau, C.; Dixneuf, P. H. Chem. Rev. 2012, 112, 5879. (c) Zhang, C.; Tang, C.; Jiao, N. Chem. Soc. Rev. 2012, 41, 3464. (d) Hickman, A. J.; Sanford, M. S. Nature 2012, 484, 177. (e) Sun, C. L.; Li, B. J.; Shi, J. Z. Chem. Rev. 2011, 111, 1293. (f) Ma, Y.; Li, W.; Yu, B. Acta Chim. Sinica 2013, 71, 541. (马玉勇, 李微, 俞蓫, 化学学报, 2013, 71, 541.) (g) Xu, J.; Chen, P.; Ye, J.; Liu, G. Acta Chim. Sinica 2015, 73, 1294. (徐佳斌, 陈品红, 叶金星, 刘国 生, 化学学报, 2015, 73, 1294.) (h) Zhang, Q.; Lü, Y.; Li, Y.; Xiong, T.; Zhang, Q. Acta Chim. Sinica 2014, 72, 1139. (张茜, 吕允贺, 李 燕, 熊涛, 张前, 化学学报, 2014, 72, 1139.) (i) Cai, H.; Li, D.; Liu, Z.; Wang, G. Acta Chim. Sinica 2013, 71, 717.

[3] Gunay, A.; Theopold, K. H. Chem. Rev. 2010, 110, 1060.

[4] Kuninobu, Y.; Nishina, Y.; Takeuchi, T.; Takai, K. Angew. Chem., Int. Ed. 2007, 46, 6518.

[5] Zhou, B.; Chen, H.; Wang, C. J. Am. Chem. Soc. 2013, 135, 1264.

[6] He, R.; Huang, Z.-T.; Zheng, Q.-Y.; Wang, C. Angew. Chem., Int. Ed. 2014, 53, 4950.

[7] Zhou, B.; Chen, H.; Wang, C. J. Am. Chem. Soc. 2013, 135, 1264.

[8] Frisch, M. J.; Trucks, G. W.; Schlegel, H. B.; Scuseria, G. E.; Robb, M. A.; Cheeseman, J. R.; Scalmani, G.; Barone, V.; Mennucci, B.; Petersson, G. A.; Nakatsuji, H.; Caricato, M.; Li, X.; Hratchian, H.
P.; Izmaylov, A. F.; Bloino, J.; Zheng, G.; Sonnenberg, J. L.; Hada, M.; Ehara, M.; Toyota, K.; Fukuda, R.; Hasegawa, J.; Ishida, M.; Nakajima, T.; Honda, Y.; Kitao, O.; Nakai, H.; Vreven, T.; Jr. Montgomery, J. A.; Peralta, J. E.; Ogliaro, F.; Bearpark, M.; Heyd, J. J.; Brothers, E.; Kudin, K. N.; Staroverov, V. N.; Keith, T.; Kobayashi, R.; Normand, J.; Raghavachari, K.; Rendell, A.; Burant, J. C.; Iyengar, S. S.; Tomasi, J.; Cossi, M.; Rega, N.; Millam, J. M.; Klene, M.; Knox, J. E.; Cross, J. B.; Bakken, V.; Adamo, C.; Jaramillo, J.; Gomperts, R.; Stratmann, R. E.; Yazyev, O.; Austin, A. J.; Cammi, R.; Pomelli, C.; Ochterski, J. W.; Martin, R. L.; Morokuma, K.; Zakrzewski, V. G.; Voth, G. A.; Salvador, P.; Dannenberg, J. J.; Dapprich, S.; Daniels, A. D.; Farkas, O.; Foresman, J. B.; Ortiz, J. V.; Cioslowski, J.; Fox, D. J. Gaussian 09, Revision B. 01, Gaussian, Inc., Wallingford CT, 2010.

[9] Zhao, Y.; Truhlar, D. G. J. Chem. Phys. 2006, 125, 194101

[10] Wadt, W. R.; Hay, P. J. J. Chem. Phys. 1985, 82, 284.

[11] (a) Fukui, K. Acc. Chem. Res. 1981, 14, 363. (b) Fukui, K. J. Phys. Chem. 1970, 74, 4161.

[12] Marenich, A. V.; Cramer, C. J.; Truhlar, D. G. J. Phys. Chem. B 2009, 113, 6378 .

[13] Fuentealba, P.; Preuss, H.; Stoll, H.; Vonszentpaly, L. Chem. Phys. Lett. 1982, 89, 418.

[14] (a) Lan, Y.; Liu, P.; Newman, S. G.; Lautens, M.; Houk, K. N. Chem. Sci. 2012, 3, 1987. (b) Yu, H.; Fu, Y. Chem. Eur. J. 2012, 18, 16765 (c) Ford, D. D.; Nielsen, L. P. C.; Zuend, S. J.; Musgrave, C. B.; Jacobsen, E. N. J. Am. Chem. Soc. 2013, 135, 15595. (d) Suresh, C. H.; Sayyed, F. B. J. Phys. Chem. A 2013, 117, 10455. (e) Yi, J.; Lu, X.; Sun, Y.-Y.; Xiao, B.; Liu, L. Angew. Chem., Int. Ed. 2013, 52, 12409. (f) Zhang, S. L.; Shi, L.; Ding, Y. Q. J. Am. Chem. Soc. 2011 133, 20218. (g) Proutiere, F.; Aufiero, M.; Schoenebeck, F. J. Am. Chem. Soc. 2012, 134, 606.

[15] (a) Xie, H.; Lin, Z. Organometallics 2014, 33, 892; (b) Schoenebeck, F.; Houk, K. N. J. Am. Chem. Soc. 2010, 132, 2496; (c) Ariafard, A.; Brookes, N. J.; Stanger, R.; Yates, B. F. Organometallics 2011, 30, 1340.

(Qin, X.) 